\title{
PEER REVIEW
}

Of

Chakravorty, I., Daga, S., Dave, S., Chakravorty, S., Menon, G., Bhala, N., Mehta, R., \&

Bamrah, J. (2020). An Online Survey of Healthcare Professionals in the COVID-19

Pandemic in the UK: : Perceptions of Risk Factors. Sushruta Journal of Health Policy \&

Opinion, 13(2). https://doi.org/10.38192/13.2.9

\section{Dhananjay V Raje PhD CSTAT}

Biostatistician

MDS Bio-Analytics, Nagpur, India

dv.raje@biomds.in

Submitted: 29.05.2020

Cite as: Raje, D.V. (2020) Peer review of 'An Online Survey of Healthcare Professionals in the COVID-19 Pandemic in the UK. Sushruta J Health Pol \& Opin 13(2) DOI:

10.38192/13.2.9.p1

Recommendations

Accept with minor revisions

Recommendations to the Authors

a) The topic is of immediate relevance to the current situation and there is a need to address the risk of infection associated with paramedical staff and the physicians geographically.

b) The authors have made a good attempt to capture the facilities provided to this staff and, whether they are able to conform to the PHE guidelines and their individual self-risk assessment, through a questionnaire-based survey. This could be one of those initial attempts to unveil the reality faced by the health workers while managing the patients, which may need immediate attention from policymakers.

c) The authors need to mention the design - Cross-sectional survey in the writeup. There is no mention of the non-parametric test used. They can simply mention about bivariate logistic regression was used to determine...

d) There are too many figures. The number can be reduced if there is a duplication of data in tables and figures. Please check.

e) The conclusions are pertinent to the survey. 